\title{
Observed properties of gamma-ray bursts
}

\author{
G.J. Fishman \\ Space Sciences Laboratory, NASA/Marshall Space Flight Center, Huntsville, AL 35812, U.S.A.
}

Received January 21; accepted April 28, 1999

\begin{abstract}
The observed temporal and spectral characteristics and the spatial distribution of gamma-ray bursts (GRBs) are summarized, primarily from data obtained with the BATSE experiment on the Compton Observatory. The availability of these data and the prospects for continued observations are also described.
\end{abstract}

Key words: gamma-rays: bursts

\section{Introduction}

Gamma-ray Bursts (GRBs) are a unique phenomenon. Their observed intense emission, which often dominates the high-energy sky during their brief appearance, has no parallel in all of astronomy. Combined with the fact that GRBs originate from sources at cosmological distances, gamma-ray bursts are truly astounding: they are the most luminous events known in the Universe.

This paper briefly describes some of the distinguishing observed features of GRBs, with particular emphasis on some recent observations with the BATSE experiment on the Compton Gamma-Ray Observatory. Data availability, dissemination and future operations are also discussed.

\section{GRB profiles and durations}

The wide diversity of GRB profiles has been known since their earliest observations. While several burst morphologies (e.g. smooth or spiky) are easy to identify, there are numerous gradations of these, as well as many complex forms. Figure 1 shows a group of four GRBs $(>20 \mathrm{keV})$ with no apparent fine time structures, unlike many bursts observed with BATSE. Most bursts have many "spikes" within the burst on timescales of $\sim 0.1 \mathrm{~s}$ and/or have numerous (sometimes dozens) of well-defined sub-pulses. The BATSE catalog (available at www.batse.msfc.nasa.gov) contains the largest dataset
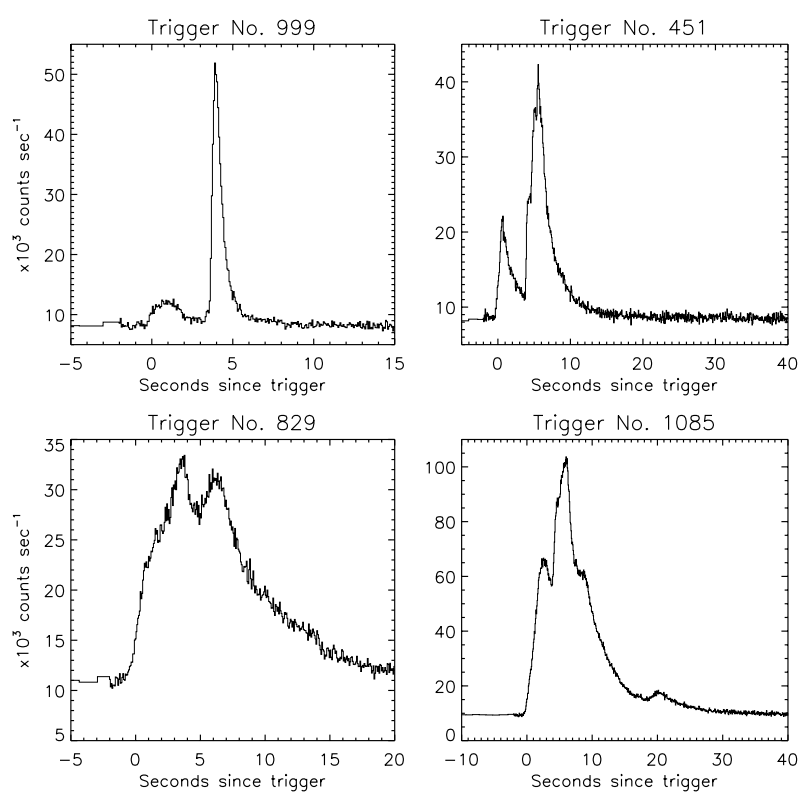

Fig. 1. Examples of some intense BATSE GRBs without fine time structures. Trigger 451 is an example of a multiple-FRED burst (Fast-Rise, Exponential Decay). In almost all multipleFREDs the rise-times of the different sub-pulses tend to be similar and much shorter than the fall-times, which also tend to be similar to each other

of GRB profiles to date. Examples of GRBs with welldefined, separated episodes of emission are also seen (Fig. 2). Attempts to quantify these structures have been largely unsuccessful. However, some of the GRB temporal features appear to be reproduced in recent models of internal shocks in GRBs (cf. Mochkovitch \& Daigne 1998; Daigne \& Mochkovitch 1998; Kobayashi et al. 1998; Beloborodov et al. 1998). Most models of the central engine of GRBs would not predict the large diversity and duration range of GRBs. An exception to this is the recent paper by Kluzniak \& Ruderman (1998) which models the central engine as a recently-formed neutron star with a high magnetic field and extreme differential 

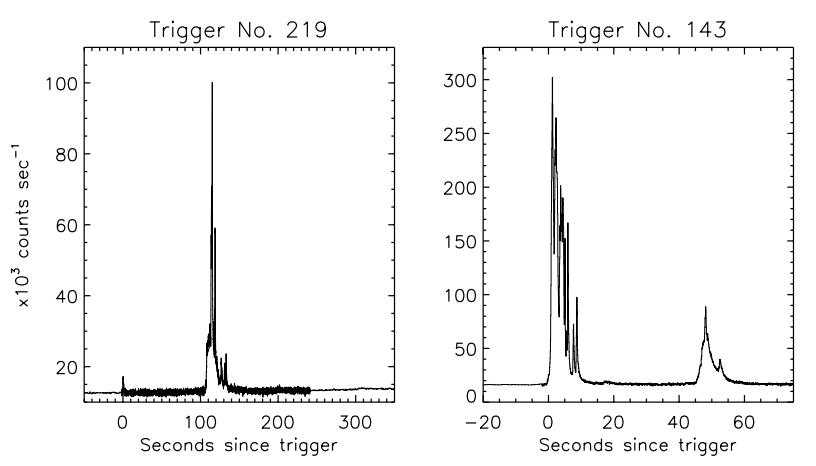

Fig. 2. Two BATSE gamma-ray bursts that have distinct, highly separated episodes of emission. In trigger 219, the onboard system triggered on a weak event lasting only a few seconds. This initial pulse was determined to come from the same location as the main emission, which followed the trigger pulse by over $100 \mathrm{~s}$

rotation. Those authors use the diversity of GRB profiles as a principal issue to be addressed by their model.

The range of the duration of gamma-ray bursts spans over five decades, from a few milliseconds to over a thousand seconds. The double peaked distribution of the duration, noted many years ago (cf. Kouveliotou et al. 1993a), is now much more evident with over two thousand observed GRBs. These two peaks in the duration distribution occur at $\sim 0.5 \mathrm{~s}$ and $\sim 34 \mathrm{~s}$. A recent statistical analysis of this distribution (Mukherjee et al. 1998) shows an indication of a third, intermediate peak (a separate class?) in this distribution. The hardness-duration correlation, which had also been described previously, is now much more evident with the large number of bursts observed with BATSE, as shown in Fig. 3.

\section{Spectra and spectral evolution}

The hard X-ray and gamma-ray spectra of GRBs have been studied in great detail (cf. papers in the 4th Huntsville GRB Symposium Proceedings, Meegan et al. 1998, and references therein). The general spectral form is well-described by what has become known as the Band function (Band et al. 1993), both in the time-integrated spectra and in shorter intervals within bursts. The low energy characteristics (below $20 \mathrm{keV}$ ) of GRBs have been examined in detail in several recent papers (Preece et al. 1996; Strohmayer et al. 1998). This is a crucial region to test emission models, such as the synchrotron shock model and the thick Comptonization model. Joint fits of spectral data from BeppoSAX and BATSE should become available in the near future.

The high-energy spectra of GRBs that are seen to extend above $10 \mathrm{MeV}$ are best fit by a power-law with a spectral index of $\sim-2$, but the number of GRBs for which these data are available are limited (cf. Schaefer

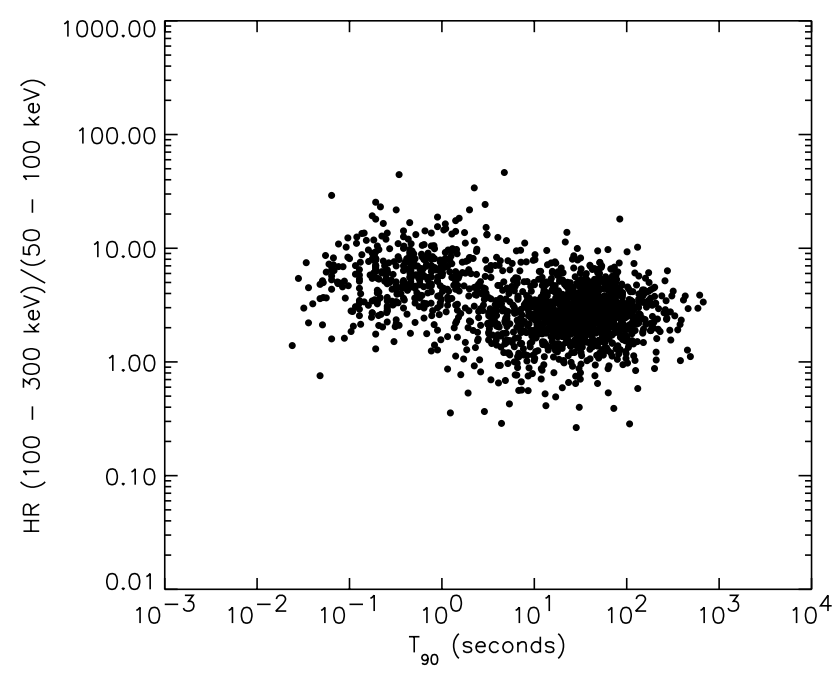

Fig. 3. The hardness-duration distribution of BATSE GRBs, showing two distinct classes of GRBs; the shorter bursts in general tend to have a harder spectrum. The Hardness Ratio (HR) is the ratio of the two middle, broad BATSE energy bands (as indicated), integrated over the duration of the GRB. The $T_{90}$ measure of the GRB duration is the standard measure used in the BATSE catalogs

et al. 1998). However, many GRB spectra are seen to cutoff much more steeply and do not have detectable emission above $300 \mathrm{keV}$ (Pendleton et al. 1997). Spectra within the BATSE range from $20 \mathrm{keV}$ to $\sim 2 \mathrm{MeV}$ have been fit to numerous GRBs (Mallozzi et al. 1995). In these spectra, both the low and high-energy ends of the broad spectral distribution are fit by smoothly joined power-laws within the BATSE bandpass. The peaks of these energy spectra $\left(\nu F_{\nu}\right)$ are referred to as the $E_{\mathrm{p}}$ energy. The $E_{\mathrm{p}}$ distribution for a large number of GRBs is shown in Fig. 4. A comparison of $E_{\mathrm{p}}$ with GRB intensity shows a correlation. This correlation is consistent with a redshift of the continuum spectrum with distance (Mallozzi et al. 1995).

Spectral softening is usually (but not always) seen throughout a GRB and also in sub-pulses within a burst. This is illustrated in Fig. 5, where a sub-peak is seen to have soft emission $(20-50 \mathrm{keV})$, for an extended duration, $\sim 8 \mathrm{~s}$, following the hard peak, which only lasts for $\sim 2$ s. In this particular case, the peaks of both the hard and soft emission occur simultaneously. However, in many cases the peaks are offset, with the softer peak delayed by up to a few seconds. The evolution of spectral properties is an active area of research. A member of the BATSE group, Giblin, is working on spectral evolution by characterizing changes and noting patterns in time-dependent color-color diagrams for ensembles of BATSE GRBs. This work is an extension of earlier color-color diagrams of BATSE GRBs (Kouveliotou et al. 1993b). Liang \& Kargatis (1996) have noted a correlation between the spectral evolution of subpeaks within a given GRB and the intensity of these subpeaks. Spectral evolution within GRBs is another property 


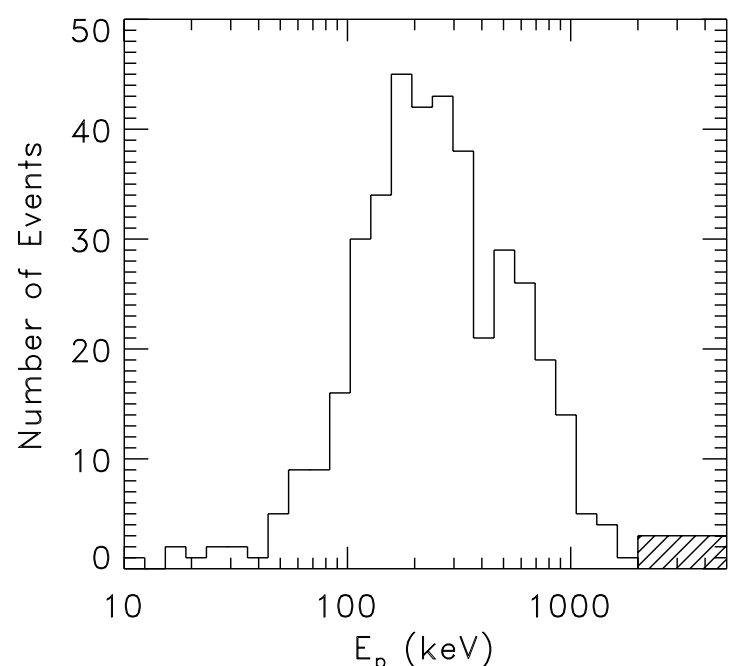

Fig. 4. The BATSE GRB $E_{\mathrm{p}}$ distribution (Mallozzi et al. 1995)

that must be considered in burst emission models but is usually not, in contrast to the well-modeled spectral evolution of the GRB afterglow emission.

\section{Sky distribution, untriggered GRBs, and BATSE location accuracy}

Figure 6 shows a recent sky distribution of nearly 2300 GRBs observed with BATSE. The statistics are now such that the effect of earth-blockage is becoming apparent on the map. This shows as a slight deficiency along the celestial equator. Corrections for this blockage yield a map with a distribution consistent with isotropy.

Efforts have been made to both improve the location accuracy of bursts observed with BATSE, and to quantify this accuracy. A recent paper by Briggs et al. (1999), shows that the BATSE systematic error, as determined by a comparison with the Interplanetary Network (IPN) GRB locations, is best described by two near-Gaussian components. The larger component has a standard deviation of $\sim 1.8 \mathrm{deg}$.

A number of recent studies have used BATSE untriggered data to search for weak events in order to extend analyses (e.g. the peak flux distribution) to weaker GRBs, to verify the BATSE on-board burst trigger, and to identify other types of transient phenomena (Kommers et al. 1997; Kommers 1999; Kommers et al. 1999; Schmidt 1999; Stern et al. 1999). These studies appear to be capable of extending the BATSE sensitivity beyond that of the triggered GRBs. However, it is expected that weak events found in these searches may be contaminated by non-GRB fluctuations of the background, hard X-ray transients, and systematic effects. It is difficult to quantify the number of the false GRBs thus found and to accurately locate them.

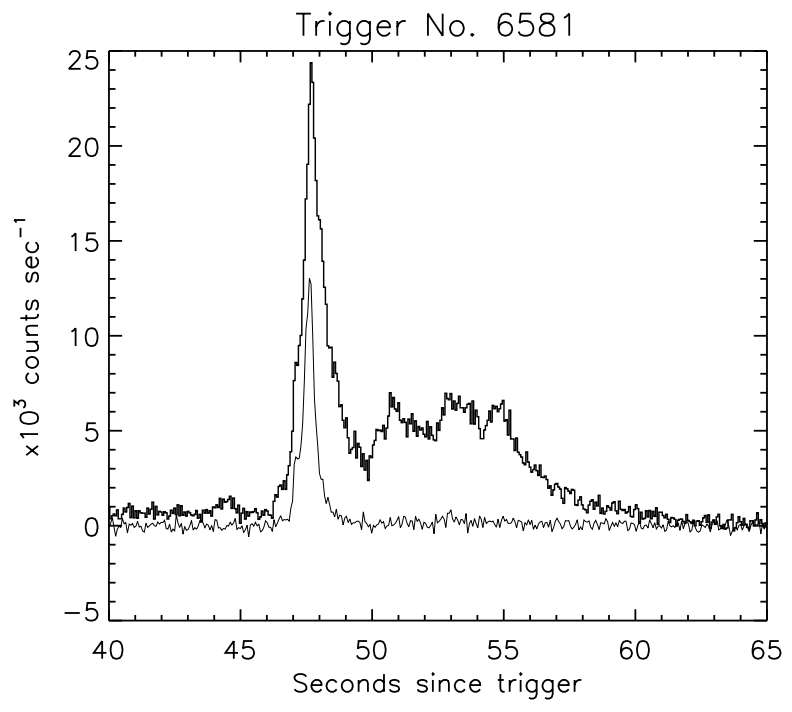

Fig. 5. An example of the hard-to-soft spectral evolution seen in many GRBs and many sub-pulses within bursts. The upper (bold) trace is time profile of the soft photons $(25-50 \mathrm{keV})$, while the lower trace shows the profile of the hardest photons observed with the BATSE large-area detectors. This hard profile of the sub-pulse shows no detectable emission at later times after this sub-pulse. The pulse itself has a width (FWHM) less than half of the softer sub-pulse

\section{GRB 980425 (=SN 1998bw?)}

During the past year, there has been considerable discussion regarding the association of GRB 980425 with the unusual supernova SN 1998bw. If the physical association is real it would provide an example of a very under-luminous (or mis-aligned, if strong beaming is assumed) gamma-ray burst, as well as a better understanding of the relationship of these two explosive phenomena. In the BATSE data, GRB 980425 does not appear to be unusual in any sense. The observed peak photon flux, $1.25 \mathrm{ph} \mathrm{cm}^{-2} \mathrm{~s}^{-1}$ (50 to $\left.300 \mathrm{keV}\right)$, is $\sim 30 \times$ less than the largest peak flux seen with BATSE from a GRB. The burst duration, as measured by the BATSE $T_{90}$ characterization (Kouveliotou et al. 1993a), is $23 \mathrm{~s}$. The morphology of this burst is a single smooth peak; this shape has been compared to many others observed with BATSE (Bonnell et al. 1999). GRB 980425 has a soft spectrum, with very little emission above $300 \mathrm{keV}$ and an $E_{\mathrm{p}} \sim$ $200 \mathrm{keV}$. This type of spectrum is observed in $\sim 20 \%$ of GRBs.

\section{BATSE operations; GRB data availability}

Gamma-ray burst data obtained with BATSE are available on three timescales in order to serve different users. GRB location information and selected ancillary GRB data are made available in near realtime and distributed 


\subsection{Years of BATSE Observations: 2292 GRBs}

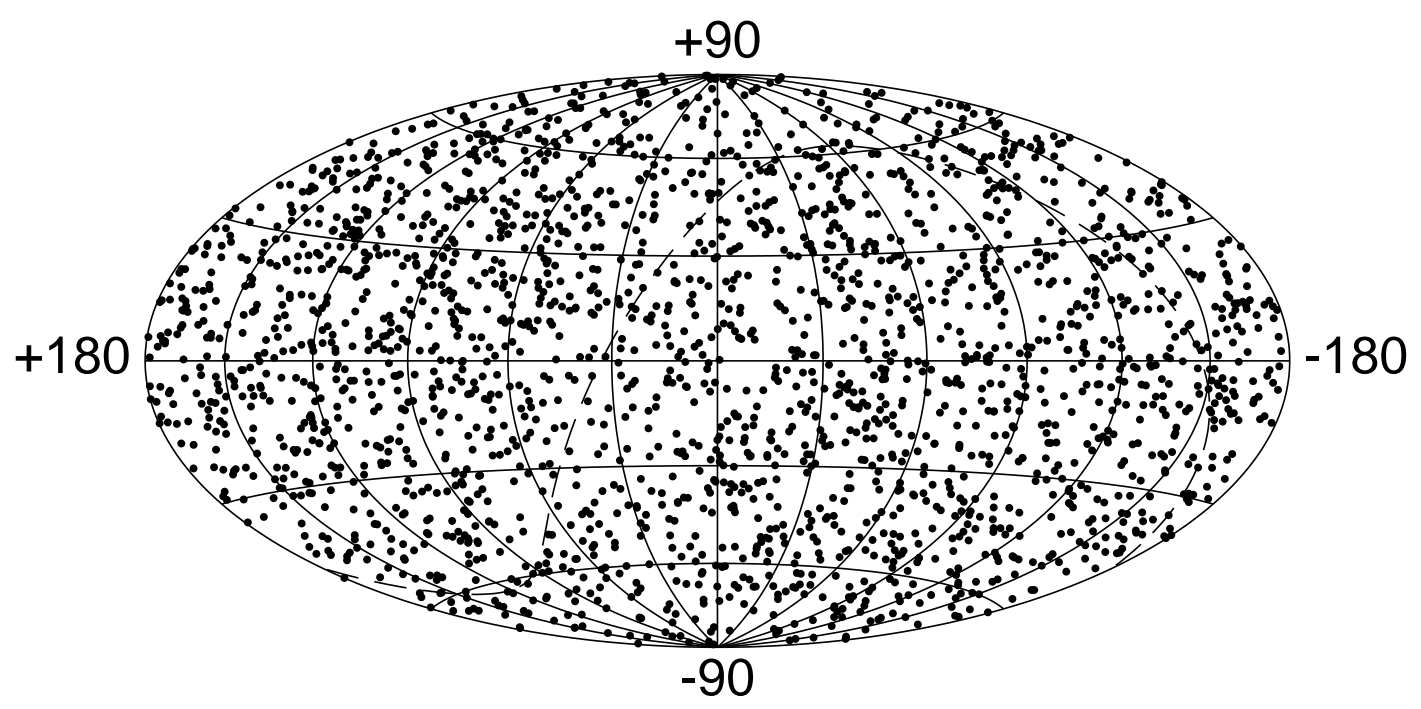

Fig. 6. The sky distribution shown in galactic coordinates of 2292 GRBs observed with BATSE. from over 7.7 years in operation. The celestial equator is shown as a long, curved dashed line. A slight deficiency of GRBs is seen in a broad region around this line due to the effects of Earth blockage

by the GCN (formerly BACODINE) network system (Barthelmy et al. 1999). These data are used by a number of robotic telescope systems currently in operation and under development. The BATSE Rapid Burst Response (RBR) locations are generated by on-call BATSE personnel in Huntsville who manually process data from selected intense GRBs. A typical location accuracy of $\sim 2$ degrees is obtained for a strong burst, significantly better than that possible with the automated BACODINE system. These RBR locations are only performed about twice per week, for the stronger bursts. The derived locations are usually processed within twenty minutes and are distributed via the GCN network.

The most comprehensive set of BATSE GRB data are in the periodically updated BATSE catalog now available on the Web. After eight years of operation, the BATSE GRB data set continues to provide the major fraction of GRB data in the energy range from $\sim 20 \mathrm{keV}$ to $\sim 2 \mathrm{MeV}$. Most data, including time profiles, are available within several days of their occurrence. It is anticipated that BATSE and the Compton Observatory will remain operational for at least another five years.

Acknowledgements. The author is grateful to T. Giblin for his help in preparing figures and the manuscript.

\section{References}

Band D., Matteson J., Ford L., et al., 1993, ApJ 413, 281

Barthelmy S., et al., 1999 (this volume)
Beloborodov A., Stern B., Svensson R., 1998, ApJ 508, L25 Bonnell J., Norris J., Wanatabe K., 1999 (this volume)

Briggs M., Pendleton G., Kippen M., et al., 1999, ApJS (in press) (astro-ph 990111)

Daigne F., Mochkovitch R., 1998, MNRAS 296, 275

Kluzniak W., Ruderman M., 1998, ApJ 505, L113

Kobayashi S., Piran T., Sari R., 1998, AIP Conf. Proc. \# 428, 672

Kommers J., Lewin W., Kouveliotou C., et al., 1997, ApJ 491, 704

Kommers J., 1999, Ph.D Thesis

Kommers J., Lewin W., Kouveliotou C., et al., 1999, ApJ (in press) (astro-ph 9809300)

Kouveliotou C., Meegan C., Fishman G., et al., 1993a, ApJ 413, L101

Kouveliotou C., Paciesas W., Fishman G., et al., 1993b, A\&AS 97,55

Liang E., Kargatis V., 1996, Nat 381, 49

Mallozzi R., Paciesas W., Pendleton G., et al., 1995, ApJ 454, 597

Meegan C., Preece R., Koshut T., 1998, AIP Conf. Proc. \# 428

Mochkovitch R., Daigne F., 1998, AIP Conf. Proc. \# 428, 667 Mukherjee S., Feigelson E., Babu J., et al., 1998, ApJ 508, 314

Pendleton G., Paciesas W., Briggs M., et al., 1997, ApJ 489, 175

Preece R., Briggs M., Pendleton G., Paciesas W., et al., 1996, ApJ 473, 310

Preece R., Briggs M., Mallozzi M., et al., 1998, ApJ 506, L23

Schaefer B., Palmer D., Dingus B., et al., 1998, ApJ 492, 696

Schmidt M., 1999 (this volume)

Stern B., et al., 1999 (this volume)

Strohmayer T., Fenimore E., Murikami T., Yoshida A., 1998, ApJ 500, 8734 